\section{CPC-029 CHARACTERIZATION OF PATIENTS NON-ADHERENT TO HIGHLY ACTIVE ANTIRETROVIRAL THERAPY (HAART) BETWEEN 2010-2011}

doi:10.1136/ejhpharm-2013-000276.486

A Alcobia, M Martins, R Bravo, A Leandro, H Duarte, P Santos, A Lopes, F Aragão. Hospital Garcia de Orta, Pharmacy, Almada, Portugal

Background Thanks to the introduction of Highly Active Antiretroviral Therapy (HAART), a decrease has been observed in the number of hospital admissions, the incidence of opportunistic infections and mortality associated with HIV/AIDS. However, adherence to treatment continues to play a crucial role. In fact, a failure of HAART often leads to changes in laboratory parameters, development of resistant strains and the need to change treatment regimens. This study aims to analyse the impact of the failure of treatment changes on laboratory parameters and treatment regimens.

Purpose To characterise patients who had been included in the government's subsidised programme who were later excluded by their inability to follow treatment.

Materials and Methods Retrospective observational study. 50 patients, who had been taking HAART since January 2007 and abandoned it between January 2010 and December 2011, were randomly selected and analysed. The control group consisted of 50 adherent patients who began taking HAART since January 2007.

Results Preliminary data indicate that our patients are mainly male with a median age range of 40 years old. There was a need to change the treatment regimen in $83 \%$ of the patients due to their inability to follow treatment correctly. Even though the viral load was undetectable in $54 \%$ of the patients, $70 \%$ couldn't achieve a CD 4 count above 350 cells/ $\mu \mathrm{L}$. The main reasons for the lack of adherence are drug and/or alcohol addiction (38\%), distance to the hospital (13\%), psychological causes (13\%), adverse drug reactions $(13 \%)$ and unknown reasons $(25 \%)$.

Conclusions The patients' inability to follow HAART correctly often leads to the need to change the treatment regimen. Considering the present data, it's extremely relevant to characterise the nonadherent population, in order to improve adherence to HAART.

No conflict of interest.

\section{CPC-030 CLINICAL CASE OF AN ADVERSE DRUG REACTION DUE TO THE ADMINISTRATION OF AN ESTROGENE/GESTAGENE COMBINATION DRUG}

doi:10.1136/ejhpharm-2013-000276.487

${ }^{1 T}$ Federica, ${ }^{1} \mathrm{~F}$ Ruggiero, ${ }^{1} \mathrm{~F}$ Borin, ${ }^{2} \mathrm{D}$ Casnaghi, ${ }^{2} \mathrm{M}$ Merlo, ${ }^{2} \mathrm{G}$ Trifirò, ${ }^{3} \mathrm{M}$ Viganò. ${ }^{1}$ A. O. "Guido Salvini", U.S.C. Farmacia, Rho, Italy; ${ }^{2}$ A. O. "Guido Salvini", U. S.C. Pediatria, Rho, Italy; ${ }^{3}$ A. O. "Guido Salvini", U.S.C. Farmacia, Garbagnate Milanese, Italy

Background Different European countries have different Summary of Product Characteristics (SmCP) for the same registered drug, which leads to an unequal level of safety information.

Purpose To describe an ADR in a child after administration of Fedra (gestodene + ethinylestradiol) not reported in the Italian SmCP case report.

Materials and Methods The Paediatric Emergency department of our hospital admitted a 15-year-old girl with frequent hand, foot and facial paresthesias on the right side of the body.

The patient had been treated with Fedra for 10 days due to menstrual irregularity.

Results At admission, the general status of the patient, monitored by blood, clotting test and imaging studies (CT and brain MRI) appeared within the normal. During the hospitalisation oestro-progestin treatment was suspended with gradual resolution of symptoms and negative neurological follow-up.

A report of these ADRs has been submitted to the Agenzia Italiana del Farmaco (AIFA) and to the database of MEAP project (monitoring adverse drug reactions in paediatric patients).

Applying the Naranjo algorithm this ADR (frequent hand, foot and facial paresthesias of the right side of the body) was classified as 'possible'.

However, in the AIFA database there are 33 ADRs related to the administration of the combo 'gestodene + ethinylestradiol' related to the nervous system, including paresthesias.

These reactions are not mentioned in the Italian SmCP, when in the British SmCP they are classified as 'well-known side effect' for the same combination, registered under the trade name of Triminulet.

Conclusions This case study suggests the importance of pointing out all the symptoms, even the minor ones not yet known, especially in the paediatric patients, to better evaluate the safety profile of the drug in this particular population.

No conflict of interest.

\section{CPC-031 CLINICAL TRIALS QUALITY: MEDICAL JOURNAL PUBLICATION INVOLVEMENT}

doi:10.1136/ejhpharm-2013-000276.488

B López García, S Ortonobes Roig, M Espona Quer, M Florit Sureda, M Barrantes Gonzalez, E Salas. Hospital del Mar, Pharmacy, Barcelona, Spain

Background Clinical Trials (CTs) resulting in publication in biomedical journals is a strategy to guarantee good quality research. Clinical research promoters are obligated to publish either positive or negative results; regretfully this good practise is not as common as it should be. Cardiology and Oncology boards are the two services carrying on the highest number of CTs in our hospital.

Purpose To cheque the CT publication index carried out by Cardiology and Oncology Clinical Services, to analyse the journals' impact factor (IP) where CTs have been published. Finally we evaluated if the published results were positive or negative.

Materials and Methods A retrospective observational study has been carried out considering all data within the period from 2002 and 2007, in the pharmacy CT unit. The study only assessed open clinical trials of Cardiology and Oncology services.

A systematic search was performed of medical journals indexed in PubMed and Clinicaltrials.gov databases until Oct. 2012.

The following variables were collected for each CT: code, journal, publication data, IP and CT results. Every CT not proving the hypothesis described in the initial protocol was considered to be a negative result (NR).

Results 229 CTs were analysed. CT/medical specialty: oncology $168(73.4 \%)$, cardiology 61 (26.6\%). CTs already closed: Oncology 85 CTs $(50.6 \%)$, cardiology 46 (75.4\%). CTs already published: 61 (26.4\%), oncology 22 (36.1\%), cardiology 39 (63.9\%). CTs published with positive results: (43 CTs) $70.5 \%$. Oncology 17 (77.3\%), Cardiology 26 (66.7\%). CTs were published in journals with IP between 2.022 and 53.298. Mean IP was 18.479 (17.406 SD).

Conclusions Fewer than $26.4 \%$ of clinical trials initiated in the two Medical Services have been published.

A high percentage of CTs initiated have never been published. Only $29.5 \%$ of all published CTs were published with a NR. This may suggest a low level of NRs result in journal publication.

The Publication IP ranges widely between low and high scores. Nevertheless we consider the mean IP to represent a high standard of publication.

No conflict of interest. 


\section{Corrections}

$\mathrm{T}$ Federica, $\mathrm{F}$ Ruggiero, $\mathrm{F}$ Borin, et al. Clinical Case of an Adverse Drug Reaction Due to the Administration of an Estrogene/Gestagene Combination Drug. Eur J Hosp Pharm 2013;20(Suppl 1): A176. doi:10.1136/ejhpharm-2013-000276. 487. The name $\mathrm{T}$ Federica was printed incorrectly. The correct name is F Taurasi.

Eur J Hosp Pharm 2013;20:204.

doi:10.1136/ejhpharm-2013-000276.487corr1 